The University of San Francisco

USF Scholarship: a digital repository @ Gleeson Library |

Geschke Center

Psychology

College of Arts and Sciences

2007

\title{
Contrasting Roles of Neural Firing Rate and Local Field Potentials in Human Memory
}

\author{
A. Ekstrom \\ Indre Viskontas \\ University of San Francisco, ivviskontas@usfca.edu \\ M. Kahana \\ J.Jacobs \\ K. Upchurch \\ See next page for additional authors
}

Follow this and additional works at: http://repository.usfca.edu/psyc

Part of the Neurology Commons, and the Psychology Commons

\section{Recommended Citation}

Ekstrom, A., Viskontas, I., Kahana, M., Jacobs, J., Upchurch, K., Bookheimer, S., Fried, I. Contrasting roles of neural firing rate and local field potentials in human memory (2007) Hippocampus, 17 (8), pp. 606-617. https://doi.org/10.1002/hipo.20300

This Article is brought to you for free and open access by the College of Arts and Sciences at USF Scholarship: a digital repository @ Gleeson Library | Geschke Center. It has been accepted for inclusion in Psychology by an authorized administrator of USF Scholarship: a digital repository@ Gleeson Library | Geschke Center. For more information, please contact repository@usfca.edu. 
Authors

A. Ekstrom, Indre Viskontas, M. Kahana, J. Jacobs, K. Upchurch, S. Bookheimer, and I. Fried 


\title{
Contrasting Roles of Neural Firing Rate and Local Field Potentials in Human Memory
}

\author{
Arne Ekstrom ${ }^{1},{ }^{*}$, Indre Viskontas ${ }^{3}$, Michael Kahana ${ }^{4}$, Josh Jacobs $^{5}$, Kristen Upchurch $^{2}$, \\ Susan Bookheimer ${ }^{1}$, and Itzhak Fried ${ }^{2}$ \\ ${ }^{1}$ Department of Psychiatry and Biobehavioral Sciences, Center for Cognitive Neurosciences, \\ Semel Institute, UCLA School of Medicine, Los Angeles, California \\ ${ }^{2}$ Department of Neurosurgery and Biobehavioral Sciences, University of California, Los Angeles, \\ California \\ ${ }^{3}$ Department of Psychology, University of California, Los Angeles, California \\ ${ }^{4}$ Department of Psychology, University of Pennsylvania, Philadelphia, California \\ ${ }^{5}$ Department of Neuroscience, University of Pennsylvania, Philadelphia, Pennsylvania
}

\begin{abstract}
Recording the activity of neurons is a mainstay of animal memory research, while human recordings are generally limited to the activity of large ensembles of cells. The relationship between ensemble activity and neural firing rate during declarative memory processes, however, remains unclear. We recorded neurons and local field potentials (LFPs) simultaneously from the same sites in the human hippocampus and entorhinal cortex (ERC) in patients with implanted intracranial electrodes during a virtual taxi-driver task that also included a memory retrieval component. Neurons increased their firing rate in response to specific passengers or landmarks both during navigation and retrieval. Although we did not find item specificity in the broadband LFP, both $\theta$ - and $\gamma$-band LFPs increased power to specific items on a small but significant percent of channels. These responses, however, did not correlate with item-specific neural responses. To contrast item-specific responses with process-specific responses during memory, we compared neural and LFP responses during encoding (navigation) and retrieval (associative and itemspecific recognition). A subset of neurons also altered firing rates nonspecifically while subjects viewed items during encoding. Interestingly, LFPs in the hippocampus and ERC increased in power nonspecifically while subjects viewed items during retrieval, more often during associative than item-recognition. Furthermore, we found no correlation between neural firing rate and broadband, $\theta$-band, and $\gamma$-band LFPs during process-specific responses. Our findings suggest that neuronal firing and ensemble activity can be dissociated during encoding, item-maintenance, and retrieval in the human hippocampal area, likely relating to functional properties unique to this region.
\end{abstract}

\section{Keywords}

hippocampus; depth electrode; local field potentials; single neuron; human memory; declarative memory

\footnotetext{
(C) 2007 Wiley-Liss, Inc.

*Correspondence to: Arne Ekstrom, UCLA Brainmapping Center, 660 Charles Young Dr., Los Angeles, CA 90095. ekstrom@ucla.edu.

This article contains supplementary material available via the Internet at http://www.interscience.wiley.com/jpages/1050-9631/ suppmat.
} 


\section{INTRODUCTION}

Differentiating the role of single neurons from ensemble activity is a particularly important issue in memory studies. Neurons demonstrate stimulus selectivity in the hippocampal area (O'Keefe and Dostrovsky, 1971), a region critical for human memory (Scoville and Milner, 1957; Vargha-Khadem et al., 1997). Furthermore, studies have also shown that neurons in the hippocampus code stimuli "sparsely," such that relatively few neurons are active at any given time during behavior (Marr, 1971; McNaughton and Morris, 1987; Waydo et al., 2006). In contrast, local field potentials (LFPs), a measure of summed neural signal, respond during more gross aspects of memory performance and exhibit less stimulus specificity in these same brain regions (Fernandez et al., 1999; Buzsaki, 2005). Although simultaneous single neuron and LFP recordings are possible in the rodent and primate via invasive electrodes, neurophysiological studies of human memory generally rely on ensemble recordings using functional magnetic resonance imaging (fMRI) or EEG at the scalp. The fMRI BOLD signal, however, may have a variable relationship with neural firing rate and LFPs (Logothetis et al., 2001; Huettel et al., 2004), correlating more strongly with LFPs than neural firing rate under some conditions (Logothetis et al., 2001; Logothetis, 2003). To investigate the relationship between ensemble and neuron activity during memory processes, we chose a paradigm studied in both humans and rodents with fMRI, LFPs, and single neurons: spatial navigation and the representation of spatial landmarks (Aguirre et al., 1996; Burgess et al., 2001; Eichenbaum, 2004; Janzen and van Turennout, 2004; Buzsaki, 2005; McNaughton et al., 2006).

The parahippocampal cortex shows greater activation in human fMRI studies when subjects view spatial scenes compared with faces and objects (Epstein and Kanwisher, 1998; Epstein et al., 2003). To investigate the navigational relevance of parahippocampal activation, Janzen and van Turrenout (2004) had subjects explore a virtual environment in which they experienced landmarks (located at decision points) and nonlandmarks. The parahippocampal cortex showed greater activation when subjects viewed landmarks during a recognition task compared with nonlandmarks, even though both types of objects were experienced during navigation (Janzen and van Turennout, 2004). We have previously shown that neurons in the (ERC) and posterior parahippocampal gyrus increase firing when patients implanted with intracranial depth electrodes viewed specific landmarks during spatial navigation (Ekstrom et al., 2003). We could not determine, however, whether the same neurons that responded to landmarks during navigation continued to fire selectively for landmarks during retrieval. Further, because we did not simultaneously record ensemble activity with neurons, we could not compare the activity of groups of neurons in the parahippocampal region (ERC and parahippocampal gyrus) with that of individual neurons. A second objective of our study, therefore, was to analyze LFP and neuron responses during viewing of spatial landmarks and following navigation during retrieval.

A final objective of our study was to simultaneously compare neural firing rate and LFPs to determine the extent to which they perform overlapping or independent computations in memory. Neural firing rate represents the activity of action potentials from individual neurons and small groups of neurons (Harris et al., 2000). LFPs, in contrast, largely represent the summation of synaptic activity - primarily excitatory postsynaptic potentials (EPSPs) - due to sinks and sources induced by movements of these currents (Nicholson, 1973; Mitzdorf, 1985). The LFP is primarily composed of low frequency activity ( $<100 \mathrm{~Hz})$ because the extracellular media acts as a low-pass filter, largely dampening the activity of action potentials, which are typically above $100 \mathrm{~Hz}$ (Bedard et al., 2004). The degree to which neural firing rate and LFPs overlap in a brain region therefore may relate to the degree to which synaptic input and action potentials are coordinated. Estimates of the 
relation between cellular firing rate and LFPs suggest some regional variability, to be expected based on cellular morphology, arrangement of inputs, and anatomical layout of neurons that differ between regions (Mitzdorf, 1985). For example, $\gamma$-band activity and neural firing rate appear to be strongly coupled in mouse visual cortex (Nase et al., 2003) and human auditory cortex (Mukamel et al., 2005) during behavior, although the correlation between LFPs and neural firing rate in primate infero-temporal cortex is significantly lower (Kreiman et al., 2006). While previous studies demonstrated phase locking between single neurons and $\gamma$-band activity in the rodent hippocampus (Bragin et al., 1995), it is not known whether behaviorally-induced increases in neural firing rate are accompanied by increases in LFP power-particularly during memory encoding and retrieval. By recording under two different behavioral conditions: self-paced navigation and timed stimulus recognition, we explored different conditions that could dissociate LFPs and neural firing rate.

\section{MATERIALS AND METHODS}

\section{Behavioral Methods}

Subjects first navigated a virtual-reality environment (VR) as if they were a taxi-driver by picking up passengers and delivering them to specific stores using our previously reported VR paradigm “yellowcab" (Ekstrom et al., 2003, 2005; Fig. 1a). Subjects navigated a $3 \times 3$ block city composed of three stores and six nontask relevant buildings. Subjects searched for passengers and delivered passengers to stores they were explicitly instructed to find within the city. Passengers were placed randomly throughout the city after each successful delivery. When a subject picked up a passenger, the passenger's picture appeared on the screen for $2 \mathrm{~s}$ with instructions of which store to find. When subjects dropped off a passenger, the passenger's picture appeared again for $2 \mathrm{~s}$. Subjects delivered nine different passengers to the three different stores. The methods described here for navigation in VR are similar to those previously described in Ekstrom et al. (2003) except (a) unique passenger identities were employed so that responses to specific passengers could be compared with responses to specific stores (b) subjects were explicitly instructed to remember which stores and passengers they met, what passengers were delivered to which stores, and the order that passengers appeared in the game.

Subjects then performed two different retrieval tasks: in the item recognition condition (Fig. 1b), subjects were presented with previously-seen and novel (lure) pictures of passengers and stores and indicated with a keypress whether or not a stimulus was seen during navigation. During the associative recognition condition (Fig. 1c) subjects viewed pairs of stimuli; either (a) a passenger and a store or (b) two passengers side-by-side. Subjects indicated (a) whether they delivered the passenger to that store or (b) which of the passengers was encountered first during navigation. The associative recognition conditions provided additional experimental conditions under which subjects viewed the same stimuli. Because the primary focus of this paper was on item vs. encoding/retrieval processes, we do not discuss or present the details of these two conditions further, referring to the three retrieval conditions as the memory condition. Some of the conditions employed in the navigation and memory conditions are similar to those used previously in an fMRI study by Burgess et al. (Burgess et al., 2001).

During the memory condition, a total of 19 items (13 passengers and six stores) were shown to subjects. Three novel stores and four novel passengers were presented during the recognition block so that not all items presented during the recognition condition would be familiar. Each stimulus or pair of stimuli was presented three times during each condition; each stimulus appeared on the screen for $3 \mathrm{~s}$ followed by a jittered interstimulus interval period of $1 \mathrm{~s} \pm 500 \mathrm{~ms}$. When patients performed a second session, they were tested in a novel spatial environment with a novel arrangement of stores and passengers. All patients 
who participated provided informed consent. All studies were conformed with the guidelines of the Medical Institutional Review Board at UCLA.

\section{Electrophysiological Methods}

Patients were implanted with depth electrodes for seizure monitoring and subsequent resection planning; all surgeries were performed by I.F. At the tip of each depth electrode a set of eight $40-\mu \mathrm{m}$ platinum-iridium microwires provided possible cellular and LFP signals (with a ninth wire distal to the recording site serving as reference). Anatomical locations of electrodes were verified by fusing CT scans taken while electrodes were implanted with high resolution MRI scans taken immediately before implantation (Fig. 1d). Signals from each microwire were amplified (gain $=10,000$ ), digitally sampled at $27.8 \mathrm{kHz}$, and bandpass filtered between $1 \mathrm{~Hz}$ and $6 \mathrm{kHz}$ (Neuralynx, Tucson, AZ). Action potentials were isolated using wave_clus. Neural events were first thresholded using the raw filtered data and then clustered based on separation of wavelet coefficients (Quiroga et al., 2004, 2005). All clusters were re-sorted manually to ensure maximum separation of different waveforms; clusters that showed contamination from $60 \mathrm{~Hz}$ activity were discarded. LFP signals were resampled at $256 \mathrm{~Hz}$ and bandpass filtered between 1 and $100 \mathrm{~Hz}$. We used the $1-100 \mathrm{~Hz}$ band for our assessment of selectivity of hippocampal area LFPs because computational estimates suggest this frequency band contains primarily synaptic activity and is largely free of action potentials (Mitzdorf, 1985; Bedard et al., 2004). We also investigated responses to the same behavioral variables in the $\theta(4-8 \mathrm{~Hz}$ in human) and $\gamma(30-100 \mathrm{~Hz})$ ranges. A 60 $\mathrm{Hz}$ notch filter (59.9-60.9 Hz stop filter constructed in Matlab) was applied to remove noise contamination from power lines. Responses that were identical on neighboring channels were not considered in the analysis.

\section{Analysis}

We identified significant responses using 19 (item) $\times 2$ (experimental context) Analysis of Variance (ANOVA) on the mean log transformed neural firing rate and mean log transformed LFP power, where "items" represented specific stimuli (e.g., a specific passenger or store) and "experimental context" represented the behavioral task the subject performed (e.g., encoding or retrieval). We thus employed similar methods to those used previously by Ekstrom et al. to detect differences in neural firing rate for landmarks during navigation (Ekstrom et al., 2003). We deemed "item" responses those that showed main effects of item and no interaction effect with experimental context because these responses would be constant across the different retrieval conditions. We deemed "experimental context" responses those that showed main effects of task but no main effect of items because we wished to study responses that were specific to navigation or retrieval and not influenced by specific items. All navigation sequences were replayed using software written in Matlab such that the passenger or store the subject was viewing during navigation was reconstructed during the subject's entire navigational trajectory. To attempt to equate the number of passenger and store viewings during retrieval, the store or passenger on the right side of the screen was considered the item being viewed during associative recognition. When we compared with viewing the objects on the left side of the screen during the associative recognition, however, our results did not change in terms of the total number of neurons or LFPs responding during item or experimental context conditions.

\section{RESULTS}

A total of 10 patients with implanted depth electrodes were tested in our paradigm. Three of these patients were unable to complete the task satisfactorily, e.g., did not complete the three retrieval conditions or did not perform above 50\% averaged across conditions. Thus, we report data from seven patients and nine recording sessions total; patients performed above 
chance during item recognition ( $85 \%$ hit rate: $t(8)=15, P<0.000001$; degrees of freedom based on nine recording sessions) and associative recognition (61\% hit rate: $t(8)=3.1, P<$ 0.01 ). We recorded and isolated a total of 119 neurons in the MTL; 36 were in the hippocampus, 57 in ERC, and 26 in the amygdala. We selected LFP channels based on sites from which we recorded neurons; we thus report from 28 hippocampal, $42 \mathrm{ERC}$, and 17 amygdala LFP recording sites.

\section{Landmark-Responsive Neurons in ERC During Navigation and Retrieval}

Previous data have shown that human MTL neurons respond to specific stimuli such as pictures of famous people or unfamiliar faces and places (Heit et al., 1988; Cameron et al., 2001; Quiroga et al., 2005). Given that our task required subjects to encode and maintain representations of stimuli across navigation and different retrieval tasks, we first determined whether neurons and LFPs responded in an item-specific manner during different experimental contexts. We did this by first binning neural firing rate and LFP wavelettransformed power (Kreiman et al., 2006) while subjects viewed an item (passenger or store) during navigation and retrieval during a 300-1,500 msec time window poststimulus, based on our previous findings of MTL neural responses during memory tasks (Viskontas et al., 2006). We considered neurons or LFPs to be item-responsive when we observed significant differences to viewing different passengers or stores ("items") in an ANOVA $(P<0.05$; see methods) across navigation and the memory condition (see Methods).

Neurons in the MTL responded with item-specific changes during navigation and also during the memory condition. In representative examples (Fig. 2a,c,e; raw traces in Supplementary Fig. 1), neurons showed increased firing rates when subjects viewed passengers and stores during both the navigation and retrieval. Over the population of MTL neurons from which we recorded, 37\% (44/119) of neurons showed significant item-related changes in firing rate throughout navigation and the memory condition (Table 1). We then subjected each item-responsive neuron to a conservative post-hoc test $(P<0.05$, corrected) in which we compared the firing rate to a specific item against the firing rate for all other items for that item-responsive neuron. Seventy-seven percent (34/44) of item-responsive neurons passed this stricter criterion (Fig. 3a); we deemed these responses "item-specific." The majority (33/34) of these neurons increased or decreased firing to only one or two items (for a breakdown of increasing and decreasing item-specific responses, Table 2). Thus, the majority of our item-responsive neurons during navigation and retrieval were selective, such that a given item-specific neuron fired to few of the stimuli presented. Although itemspecific neurons were evenly distributed across the MTL, we found a greater number itemspecific neurons changing firing rate to stores in ERC compared with hippocampus and amygdala (Chi square test, $\chi^{2}(2)=6.2, P<0.05$, Fig. $3 b$ ). The clustering of landmarkresponsive neurons in the ERC during navigation and retrieval comports well with our previous findings of landmark responsive neurons clustering in the parahipocampal region (ERC and parahippocampal gyrus) during navigation (Ekstrom et al., 2003).

We then performed identical analyses with our simultaneously recorded LFPs to look for changes in LFP power to specific items during navigation and retrieval. In contrast to neurons, broadband LFPs did not show significant selectivity for items (Fig. 2b,d,f; raw traces in Supplementary Fig. 1); only 4\% (3/87) of channels with LFP signal responded significantly to a particular item, which did not exceed the Type I error rate $\left(\chi^{2}(1)=0.1, P=\right.$ n.s.; Fig. 3a and Table 1). Theta and $\gamma$-band LFPs, however, showed item selectivity compared with the broadband LFP (Fig. 3a and Table 1). The $\theta$-band LFP modulated power to specific items in 13\% (11/87) of channels while $\gamma$-band LFP modulated power to specific items in $17 \%(15 / 87)$ of channels. Both of these responses exceeded the type I error rate ( $\theta$ :

$\left.\chi^{2}(1)=7.2, P<0.01 ; \gamma: \chi^{2}(1)=28, P<0.00001\right)$. The responses of $\theta$ - and $\gamma$-band LFPs to stores and passengers are shown in Table 2; we did not observe regional differences for 
item-specific LFP responses. Item-specific neural responses exceed item-specific $\theta$ responses (neurons vs. $\theta \chi^{2}(1)=5.3, P<0.01$ ) but not item-specific $\gamma$ responses $\left(\chi^{2}(1)=\right.$ 3.0, $P=$ n.s. $)$.

\section{LFP Responses During Retrieval}

Previous LFP recordings found greater oscillatory power during retrieval compared with encoding while patients with implanted electrodes navigated a virtual environment (Kahana et al., 1999). Because our experiment involved encoding during navigation and retrieval of these items following navigation, we hypothesized that we might also observe changes in LFPs or firing rate for the different experimental contexts that were not specific to items. LFPs that showed a significant effect of experimental context (navigation vs. retrieval but not to items; see methods) were then subjected to additional post-hoc tests $(P<0.05$, corrected) in which LFP power during a specific experimental context (navigation, recognition, or associative recognition) was compared with LFP power during the other experimental contexts.

In representative examples, the mean LFP power on MTL channels showed significant experimental context-related changes, increasing in power across frequencies during retrieval compared with navigation (Fig. 4b,d,h). Twenty percent (17/87) of broadband LFP responses showed modulations to experimental context, which significantly exceeded the Type I error rate $\left(\chi^{2}(1)=26, P<0.00001\right.$; Fig. 3a). We observed significantly more experimental context-related broadband LFP responses than broadband LFP item responses (20 vs. $4 \%$ ). Theta and $\gamma$-band LFPs responded in comparable numbers to experimental context $(\theta: 13 \% ; \gamma 12 \%$, Table 3$)$, although the number of electrodes showing $\theta$ - and $\gamma$ band LFP context responses was not significantly different from the number of electrodes showing $\theta$ - and $\gamma$-band LFP item responses (Fig. 3a, Table 3).

We then determined during which experimental context(s) LFPs responded with a change in power. The majority of broadband, $\theta$ and $\gamma$ LFP responses increased during retrieval compared with encoding (Table 3 and Fig. 3c); a small number of $\gamma$-band LFPs also responded with greater power during encoding (Table 3). In all frequency bands, however, there were significantly more electrodes active during retrieval compared with encoding (broad-band: 17 vs. $0,\left[\chi^{2}(1)=17, P<0.0001\right]$; $\theta$-band, 11 vs. $0,\left[\chi^{2}(1)=11, P<0.001\right]$; $\gamma$-band, 8 vs. $2,\left[\chi^{2}(1)=5, P<0.05\right]$. Experimental context-related LFP responses were evenly distributed throughout the hippocampus, ERC, and amygdala (Table 3). The broadband LFP, but not the $\theta$ and $\gamma$ LFP, showed greater power during associative recognition compared with item-recognition (9 LFPs were significantly greater during associative recognition compared with 1 LFP significantly greater during item-recognition $\left(\chi^{2}(1)=6.4, P<0.01\right.$, Table 3). Also, greater numbers of electrodes showed increased power during associative recognition in hippocampus and ERC compared with the amygdala $\left(\chi^{2}(1)=9, P<0.01\right)$.

The broadband LFP therefore showed the least selectivity for items (4\%) and the greatest selectivity for experimental context (20\%). Broadband LFP responses showed significantly greater amplitude during retrieval compared with navigation, and showed the greatest amplitude during associative recognition compared with item recognition. Broadband LFPs responding significantly during associative recognition were present to greater degrees in hippocampus and ERC than amygdala. While both $\gamma$ - and $\theta$-bands showed some selectivity for items (13 and 17\%), $\gamma$ - and $\theta$-bands also responded significantly to experimental context (13 and 12\%). Similar to the broadband LFP, we found the greatest number of $\theta$ and $\gamma$ LFP responses during retrieval compared with navigation. 


\section{Neural Firing Rate Changes During Encoding}

We then performed the same experimental context-related analyses we reported for LFPs on neural firing rate (see Methods). We found that 25\% (30/119) of neurons responded significantly to experimental context (Fig. 3a, Table 1). To compare our experimental context-related LFP amplitude and power changes with our experimental context-related firing rate changes, we compared neural responses with LFP responses on the same channel (Fig. 4b,d,f,h). In contrast to LFP responses, neurons often showed increased firing during navigation compared with retrieval (Fig. 4a,c,e). Post-hoc comparisons, identical to those previously performed on LFPs, revealed that the majority of these context-related neurons increased their firing rate during navigation compared with retrieval (77\% [23/30] vs. $23 \%$ [7/30]; $\chi^{2}(1)=8.5, P<0.01$; Fig. 3c). We found a significant dissociation for neurons responding during encoding and LFPs responding during retrieval. This effect was most pronounced for the broadband LFP compared with neural firing rate (neurons, encoding vs. retrieval: 23 vs. 7 , broadband LFP, 0 vs. 17, Fischer's exact test, $P<0.00001$ ) but was also significant for $\theta$-band (Fischer's exact test, $P<0.005$, Table 3 ) and $\gamma$-band LFPs (Fischer's exact test, $P<0.0001$, Table 3). Our results for experimental context-related neurons thus show the opposite trend to context-related LFPs: more experimental context-related neurons were active during encoding than retrieval.

\section{No Correlation Between Neural Firing Rate and LFPs Over Population of Responses}

Despite the dissociations we observed between neurons and LFPs for experimental context, it could still be the case that neural firing rate changes and LFP changes were closely related in time. We also wished to address whether neural firing rate and LFP power changes were coupled for item responses, because neuron, $\theta$-band LFPs, and $\gamma$-band LFPs all showed significant item-responses. If neurons and LFPs were associated, we would expect firing rate and LFP power changes to correlate on the same channels. To address this issue, we computed the correlation between the mean firing rate with the mean broadband wavelettransformed LFP power between 300 and $1,500 \mathrm{~ms}$ poststimulus using methods identical to Krieman et al. (2006). This analysis was done separately for channels showing significant changes in firing rate to items (44/119) and experimental context-selective changes in firing rate (e.g., during encoding or retrieval, 30/119). The mean correlation across the population of cellular and LFP channels did not differ from zero for items (Fig. 5; $r=-0.01 \pm 0.02$; $t(43)=-0.85 ; P=$ n.s. $)$ or experimental context $(r=-0.06 \pm 0.06, t(29)=0.73, P=$ n.s. $)$.

Although classic hippocampal LFPs are seen between 1 and $100 \mathrm{~Hz}$ (Halgren and Smith, 1987; McCarthy et al., 1995; Fernandez et al., 1999), correlations between firing rate and LFPs have typically been reported above the $\gamma$-band (Mukamel et al., 2005), e.g., 40-100 $\mathrm{Hz}$. We thus redid our population correlation analysis for LFP power separately in the $\theta$ (4(8) $\mathrm{Hz})$ and $\gamma$-band $(40-100 \mathrm{~Hz})$. Correlations between cellular firing rate for item and experimental context responses and $\theta$-band power were not significantly different from zero (Fig. 5; item: $r=-0.01 \pm 0.2, t(43)=-0.90, P=$ n.s.; context: $r=0.05 \pm 0.03, t(29)=1.8, P$ $=$ n.s.), and neither were correlations between cellular firing rate for item and experimental context responses and $\gamma$-band power (Fig. 5; item: $r=-0.02 \pm 0.05, t(43)=0.3, P=$ n.s.; context: $t(29)=-0.8, P=$ n.s). To ensure there were no regional, frequency band, or behaviorally induced differences in these correlation, we conducted a $3 \times 3 \times 2$ region (amygdala vs. hippocampus vs. ERC) $\times$ band (broadband vs. $\theta$ vs. $\gamma$ ) $\times$ behavioral variable (item vs. experimental context) ANOVA. There were no main effects of any variable and no significant interactions (see Tables 2 and 3 for correlations broken down by region). 


\section{DISCUSSION}

Our task, which required encoding of items and associations between items during navigation as well as recognition of those items and their associations, provided an opportunity to contrast the neural basis of item-related and experimental context-related representations. Our paradigm also permitted us to contrast representations formed during navigation with representations accessed during retrieval. It is thus notable that a high percent $(29 \%)$ of neurons showed changes in firing rate for viewing specific items during encoding which persisted through retrieval, despite differences in experimental context between the different tasks. These responses were typically specific to a few items, suggesting that item-specific representations are formed in the hippocampal area. While selective, item-specific neural responses have been reported previously in the MTL during viewing of famous people and other objects (Heit et al., 1988; Kreiman et al., 2000; Quiroga et al., 2005), we show here that these representations are invoked during both item-learning and during learning of episodes involving formation of item-context associations.

We also found greater numbers of neurons responding during viewing of stores in the ERC compared with the hippocampus and amygdala. We previously described neurons present in the ERC and parahippocampal gyrus (parahippocampal region) that increased firing during viewing of landmarks during navigation (Ekstrom et al., 2003). Because we did not find significant numbers of store-responsive neurons firing during a control task in which subjects viewed stores and read their names out loud prior to navigation (Ekstrom et al., 2003), our results suggest that ERC neurons were specifically involved in encoding spatial landmarks. Our current results extend our previous findings to show that neurons in the ERC increase firing rate to specific stores and maintain their firing during both simple recognition of these objects and during retrieval of these objects along with the context in which they were encoded. Thus, our results show that the same ERC neurons that encode spatial landmarks during navigation maintain their altered firing rates when these stores are viewed during retrieval.

In contrast to the significant changes in neural firing rate for items encountered during navigation and retrieval, we did not find significant numbers of item-specific broadband LFPs changing during navigation and retrieval. These data suggest that during navigation and retrieval, the broadband LFP does not convey information about specific stimuli. We did, however, observe item-specific responses in $\theta$ - and $\gamma$-band. Previous work demonstrated increased $\theta$ and $\gamma$ amplitude during encoding of items that are subsequently recalled (Sederberg et al., 2003; Mormann et al., 2005). Because we did not find a significant correlation between neural item responses and $\theta$-band and $\gamma$-band LFP responses, our results suggest that $\theta$-band and $\gamma$-band LFPs do not directly relate to neural firing rate changes.

We did observe significant LFP power changes to experimental contexts in all frequency bands. A significantly greater number of broadband, $\theta$-band, and $\gamma$-band LFPs were active while subjects viewed passengers and stores during retrieval compared with encoding. In contrast, we found significantly greater numbers of neurons active while subjects viewed stores and passengers during encoding compared with retrieval. It is notable that we again dissociated neurons and LFPs based on encoding vs. retrieval. It is possible that the selftimed nature of navigation led to a greater dissociation of LFPs during encoding (while navigating) compared with retrieval. Previous scalp EEG recordings, however, during a timed, continuous recognition task demonstrate greater ERP amplitude during retrieval compared with encoding (Finnigan et al., 2002). Also, Kahana et al. (1999) observed greater $\theta$ oscillatory power during retrieval of a spatial locations while recalling learned navigational routes than during encoding of these routes (Kahana et al., 1999). Thus, given 
that previous studies have also reported greater LFP power during retrieval compared with encoding we believe that our finding of greater broadband, $\theta$, and $\gamma$-band LFP power during retrieval compared with encoding is therefore not specific to the self-paced nature of navigation.

While we found greater numbers of electrodes active during retrieval compared with encoding in all frequency bands, we also observed greater numbers of broadband LFPs responding during associative compared with item recognition. This finding further argues that the greater broadband LFP power we observed during retrieval when compared with encoding relates to retrieval-specific processes. Because we found greater numbers of electrodes responding during associative compared with item recognition in the hippocampus and ERC compared with the amygdala, our data further argue that retrievalspecific increases in LFPs during associative retrieval may be unique to memory processing in these two regions. Given that we observed this effect in the LFP, a reflection of synaptic input (Mitzdorf, 1985; Logothetis, 2003), but not in neural firing rate, our data further suggest that retrieving associations requiring both item and source information leads to greater input of synaptic activity in the hippocampus and ERC compared with item recognition.

A final question we wished to address was the simultaneous relation between neurons and LFPs during memory processing. Previous recordings from human auditory cortex suggest a high correlation between neural firing rate and $\gamma$-band LFPs during listening to complex sounds (Mukamel et al., 2005). In the Mukamel et al. (2005) experiment, however, large numbers of neurons were simultaneously active during the task, suggesting coordinated input and output processes (Logothetis, 2003). In our experiment, few neurons responded at any given time to items due to their selectivity for certain items and not others. It is thus not surprising that we observed little or no population correlation between neurons and LFPs for items in any frequency band. Although we observed significant $\theta$ - and $\gamma$-band responses to items, cellular responses to items did not correlate with either $\theta$ - or $\gamma$-band LFPs. These data suggest that neural and $\theta / \gamma$ band LFP responses were not associated in time, and that $\theta / \gamma$ band LFP responses were to different objects at different times than cellular responses. On the basis of our dissociation for neural responses during encoding and LFP responses during retrieval, we tentatively suggest that our item-related neural responses relate to itemmaintenance, while LFP responses may relate to attentional process involved in processing specific objects in memory.

The lack of a correlation between experimental context-related neurons and LFPs is somewhat more surprising because these experimental context-related responses were active over an entire block of the experiment (e.g., during navigation or retrieval). Given, however, that neurons and LFP power (across all bands) increased during complementary phases of the experiment, with neurons increasing firing during encoding, and LFPs increasing in power during retrieval, this lack of a correlation is not surprising based on our behavioral results. In support of our finding of a dissociation between LFP power and neural firing during navigation, a recent spatial learning study in rodents by Robbe et al. (2006) similarly observed little correlation between LFP power and neural firing rate (e.g., Robbe et al., Fig. 5 ), although the authors did not look specifically at encoding or retrieval of landmarks (Robbe et al., 2006).

It is important to note here that individual neurons we recorded from may show strong coupling in the $\gamma$ frequency range even though the behaviorally-responsive population we analyzed, on average, did not. In fact, previous results suggest that subsets of neurons do in some case phase lock with the ongoing $\gamma$ oscillation in rat and human hippocampus (Bragin et al., 1995; Jacobs et al., 2007). Our findings, however, showed that over the population of 
item and experimental context responses, there was little correlation with $\gamma$ oscillatory power, a result bolstered by our behavioral findings. Because our primary interest was the relationship between the population of simultaneously recorded neurons and LFPs in the hippocampal area for comparison with previous studies (Buchwald et al., 1965; Wyler et al., 1982; Logothetis et al., 2001; Logothetis, 2003; Nase et al., 2003; Mukamel et al., 2005; Kreiman et al., 2006), we did not focus on individual neurons that showed strong coupling or anticoupling with $\gamma$. What we wish to emphasize is that the correlation over the population of neurons and LFPs in our study was significantly lower than that observed in previous studies in other brain regions, despite the similarity of our methods to previous studies. Correlations between cellular firing rate and LFPs over the population of recording sites in auditory cortex of humans and infero-temporal cortex of monkeys, particularly in the $\gamma$-band, are typically significantly higher than what was observed in this study, reported in the range of 0.2-0.8 (Mukamel et al., 2005; Kreiman et al., 2006).

The correlations reported in this study in the hippocampal area and amygdala, in contrast, did not differ significantly from zero. These data therefore suggest that the hippocampus, ERC, and amygdala have a different functional-anatomical layout than sensory regions in the brain. In support of this notion, recordings from rodent hippocampus demonstrate no correlation between neighboring neurons and their behavioral correlates (Redish et al., 2001), in contrast to the visual cortex, where neurons responding to the orientation of bars are arranged in hypercolumns based on their preferred orientation (Mountcastle, 1997). The fact that increased selectivity of neurons is often accompanied by decreases in global activity in the hippocampus (Hirase et al., 2001) further supports our finding of no significant LFP change during selective neural epochs in the hippocampal area.

Because of the behavioral dissociations for neural firing rate and LFPs, and the nonsignificant correlations we found between cellular firing rate and LFPs in the hippocampus compared with the larger correlations reported in previous studies in other brain regions (Mukamel et al., 2005; Kreiman et al., 2006), we believe the differences we observed between cellular firing rate and LFPs also in part relate to differences in what these two signals represent. LFPs likely reflect the input to a brain region because they largely represent the summation of excitatory synaptic events (e.g., primarily EPSPs) due to afferent input (Mitzdorf, 1985). Neural firing rate (e.g., action potentials), however, represents the computations and therefore the output of a region (Koch and Segev, 2000; Logothetis, 2003). Our study thus provides preliminary evidence for a distinction between input-related LFP responses and output-related spiking activity during declarative memory processes in the human hippocampus. We suggest therefore that our findings, in conjunction with previous electrophysiological studies showing stimulation-induced differences between LFP-related input and neuron-related output (Mathiesen et al., 1998, 2000; Lauritzen, 2001; Lauritzen and Gold, 2003), support distinct functional roles for ensemble activity (LFP and the fMRI BOLD signal) and cellular firing rate in the human hippocampus during declarative memory processes.

\section{Supplementary Material}

Refer to Web version on PubMed Central for supplementary material.

\section{Acknowledgments}

The authors gratefully acknowledge the participation of all patients involved in the study and Saba Moshirvaziri, Tony Fields, and Emily Ho for technical assistance. We also thank Russ Poldrack for reading and commenting on this manuscript.

Grant sponsor: NINDS; Grant number: F32 NS50067. 


\section{References}

Aguirre GK, Detre JA, Alsop DC, D’Esposito M. The parahippocampus subserves topographical learning in man. Cereb Cortex. 1996; 6:823-829. [PubMed: 8922339]

Bedard C, Kroger H, Destexhe A. Modeling extracellular field potentials and the frequency-filtering properties of extracellular space. Biophys J. 2004; 86:1829-1842. [PubMed: 14990509]

Bragin A, Jando G, Nadasdy Z, Hetke J, Wise K, Buzsaki G. Gamma (40-100 Hz) oscillation in the hippocampus of the behaving rat. J Neurosci. 1995; 15:47-60. [PubMed: 7823151]

Buchwald JS, Halas ES, Schramm S. Comparison of multiple-unit and electroencephalogram activity recorded from the same brain sites during behavioural conditioning. Nature. 1965; 205:1012-1014.

Burgess N, Maguire EA, Spiers HJ, O'Keefe J. A temporoparietal and prefrontal network for retrieving the spatial context of lifelike events. Neuroimage. 2001; 14:439-453. [PubMed: 11467917]

Buzsaki G. Theta rhythm of navigation: Link between path integration and landmark navigation, episodic and semantic memory. Hippocampus. 2005; 15:827-840. [PubMed: 16149082]

Cameron KA, Yashar S, Wilson CL, Fried I. Human hippocampal neurons predict how well word pairs will be remembered. Neuron. 2001; 30:289-298. [PubMed: 11343662]

Eichenbaum H. Hippocampus: Cognitive processes and neural representations that underlie declarative memory. Neuron. 2004; 44:109-120. [PubMed: 15450164]

Ekstrom AD, Kahana MJ, Caplan JB, Fields TA, Isham EA, Newman EL, Fried I. Cellular networks underlying human spatial navigation. Nature. 2003; 425:184-188. [PubMed: 12968182]

Ekstrom AD, Caplan JB, Ho E, Shattuck K, Fried I, Kahana MJ. Human hippocampal theta activity during virtual navigation. Hippocampus. 2005; 15:881-889. [PubMed: 16114040]

Epstein R, Kanwisher N. A cortical representation of the local visual environment. Nature. 1998; 392:598-601. [PubMed: 9560155]

Epstein R, Graham KS, Downing PE. Viewpoint-specific scene representations in human parahippocampal cortex. Neuron. 2003; 37:865-876. [PubMed: 12628176]

Fernandez G, Effern A, Grunwald T, Pezer N, Lehnertz K, Dumpelmann M, Van Roost D, Elger CE. Real-time tracking of memory formation in the human rhinal cortex and hippocampus. Science. 1999; 285:1582-1585. [PubMed: 10477525]

Finnigan S, Humphreys MS, Dennis S, Geffen G. ERP old/new effects: Memory strength and decisional factor(s). Neuropsychologia. 2002; 40:2288-2304. [PubMed: 12417459]

Halgren E, Smith ME. Cognitive evoked potentials as modulatory processes in human memory formation and retrieval. Hum Neurobiol. 1987; 6:129-139. [PubMed: 3305439]

Harris KD, Henze DA, Csicsvari J, Hirase H, Buzsaki G. Accuracy of tetrode spike separation as determined by simultaneous intracellular and extracellular measurements. J Neurophysiol. 2000; 84:401-414. [PubMed: 10899214]

Heit G, Smith ME, Halgren E. Neural encoding of individual words and faces by the human hippocampus and amygdala. Nature. 1988; 333:773-775. [PubMed: 3386719]

Hirase H, Leinekugel X, Czurko A, Csicsvari J, Buzsaki G. Firing rates of hippocampal neurons are preserved during subsequent sleep episodes and modified by novel awake experience. Proc Natl Acad Sci USA. 2001; 98:9386-9390. [PubMed: 11470910]

Huettel SA, McKeown MJ, Song AW, Hart S, Spencer DD, Allison T, McCarthy G. Linking hemodynamic and electrophysiological measures of brain activity: Evidence from functional MRI, intracranial field potentials. Cereb Cortex. 2004; 14:165-173. [PubMed: 14704213]

Jacobs J, Kahana MJ, Ekstrom AD, Fried I. Brain oscillations control timing of single neuron activity in humans. J Neurosci. 2007; 27:3839-3844. [PubMed: 17409248]

Janzen G, van Turennout M. Selective neural representation of objects relevant for navigation. Nat Neurosci. 2004; 7:673-677. [PubMed: 15146191]

Kahana MJ, Sekuler R, Caplan JB, Kirschen M, Madsen JR. Human theta oscillations exhibit task dependence during virtual maze navigation. Nature. 1999; 399:781-784. [PubMed: 10391243]

Koch C, Segev I. The role of single neurons in information processing. Nat Neurosci. 2000; 3(Suppl): 1171-1177. [PubMed: 11127834] 
Kreiman G, Koch C, Fried I. Category-specific visual responses of single neurons in the human medial temporal lobe. Nat Neurosci. 2000; 3:946-953. [PubMed: 10966627]

Kreiman G, Hung CP, Kraskov A, Quiroga RQ, Poggio T, DiCarlo JJ. Object selectivity of local field potentials and spikes in the macaque inferior temporal cortex. Neuron. 2006; 49:433-445. [PubMed: 16446146]

Lauritzen M. Relationship of spikes, synaptic activity, and local changes of cerebral blood flow. J Cereb Blood Flow Metab. 2001; 21:1367-1383. [PubMed: 11740198]

Lauritzen M, Gold L. Brain function and neurophysiological correlates of signals used in functional neuroimaging. J Neurosci. 2003; 23:3972-3980. [PubMed: 12764081]

Logothetis NK. The underpinnings of the BOLD functional magnetic resonance imaging signal. J Neurosci. 2003; 23:3963-3971. [PubMed: 12764080]

Logothetis NK, Pauls J, Augath M, Trinath T, Oeltermann A. Neurophysiological investigation of the basis of the fMRI signal. Nature. 2001; 412:150-157. [PubMed: 11449264]

Marr D. Simple memory: A theory for archicortex. R Soc Lond Biol Sci. 1971; 262:23-81.

Mathiesen C, Caesar K, Akgoren N, Lauritzen M. Modification of activity-dependent increases of cerebral blood flow by excitatory synaptic activity and spikes in rat cerebellar cortex. J Physiol. 1998; 512 (Pt 2):555-566. [PubMed: 9763643]

Mathiesen C, Caesar K, Lauritzen M. Temporal coupling between neuronal activity and blood flow in rat cerebellar cortex as indicated by field potential analysis. J Physiol. 2000; 523(Part 1):235-246. [PubMed: 10673558]

McCarthy G, Nobre AC, Bentin S, Spencer DD. Language-related field potentials in the anteriormedial temporal lobe. I. Intracranial distribution and neural generators. J Neurosci. 1995; 15:1080-1089. [PubMed: 7869084]

McNaughton BL, Morris RGM. Hippocampal synaptic enhancement and information storage within a distributed memory system. Trends Neurosci. 1987; 10:408-415.

McNaughton BL, Battaglia FP, Jensen O, Moser EI, Moser MB. Path integration and the neural basis of the cognitive map. Nat Rev Neurosci. 2006; 7:663-678. [PubMed: 16858394]

Mitzdorf U. Current source-density method and application in cat cerebral cortex: Investigation of evoked potentials and EEG phenomena. Physiol Rev. 1985; 65:37-100. [PubMed: 3880898]

Mormann F, Fell J, Axmacher N, Weber B, Lehnertz K, Elger CE, Fernandez G. Phase/amplitude reset and theta-gamma interaction in the human medial temporal lobe during a continuous word recognition memory task. Hippocampus. 2005; 15:890-900. [PubMed: 16114010]

Mountcastle VB. The columnar organization of the neocortex. Brain. 1997; 120(Part 4):701-722. [PubMed: 9153131]

Mukamel R, Gelbard H, Arieli A, Hasson U, Fried I, Malach R. Coupling between neuronal firing, field potentials, and FMRI in human auditory cortex. Science. 2005; 309:951-954. [PubMed: 16081741]

Nase G, Singer W, Monyer H, Engel AK. Features of neuronal synchrony in mouse visual cortex. J Neurophysiol. 2003; 90:1115-1123. [PubMed: 12702711]

Nicholson C. Theoretical analysis of field potentials in anisotropic ensembles of neuronal elements. IEEE Trans Biomed Eng. 1973; 20:278-288. [PubMed: 4708762]

O'Keefe J, Dostrovsky J. The hippocampus as a spatial map. Preliminary evidence from unit activity in the freely-moving rat. Brain Res. 1971; 34:171-175. [PubMed: 5124915]

Quiroga RQ, Nadasdy Z, Ben-Shaul Y. Unsupervised spike detection and sorting with wavelets and superparamagnetic clustering. Neural Comput. 2004; 16:1661-1687. [PubMed: 15228749]

Quiroga RQ, Reddy L, Kreiman G, Koch C, Fried I. Invariant visual representation by single neurons in the human brain. Nature. 2005; 435:1102-1107. [PubMed: 15973409]

Redish AD, Battaglia FP, Chawla MK, Ekstrom AD, Gerrard JL, Lipa P, Rosenzweig ES, Worley PF, Guzowski JF, McNaughton BL, Barnes CA. Independence of firing correlates of anatomically proximate hippocampal pyramidal cells. J Neurosci. 2001; 21:RC134. [PubMed: 11222672]

Robbe D, Montgomery SM, Thome A, Rueda-Orozco PE, McNaughton BL, Buzsaki G. Cannabinoids reveal importance of spike timing coordination in hippocampal function. Nat Neurosci. 2006; 9:1526-1533. [PubMed: 17115043] 
Scoville WB, Milner B. Loss of recent memory after bilateral hippocampal lesions. J Neurol Neurosurg Psychiatry. 1957; 20:11-21. [PubMed: 13406589]

Sederberg PB, Kahana MJ, Howard MW, Donner EJ, Madsen JR. Theta and gamma oscillations during encoding predict subsequent recall. J Neurosci. 2003; 23:10809-10814. [PubMed: 14645473]

Vargha-Khadem F, Gadian DG, Watkins KE, Connelly A, Van Paesschen W, Mishkin M. Differential effects of early hippocampal pathology on episodic and semantic memory. Science. 1997; 277:376-380. [PubMed: 9219696]

Viskontas I, Knowlton B, Steinmetz PN, Fried I. Differences in mnemonic processing by neurons in the human hippocampus and parahippocampal region. J Cogn Neurosci. 2006; 20:1654-1662. [PubMed: 17014370]

Waydo S, Kraskov A, Quian Quiroga R, Fried I, Koch C. Sparse representation in the human medial temporal lobe. J Neurosci. 2006; 26:10232-10234. [PubMed: 17021178]

Wyler AR, Ojemann GA, Ward AA Jr. Neurons in human epileptic cortex: Correlation between unit and EEG activity. Ann Neurol. 1982; 11:301-308. [PubMed: 7092182] 
a.

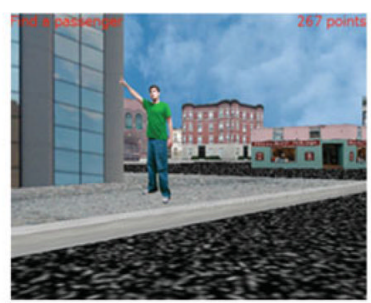

c.

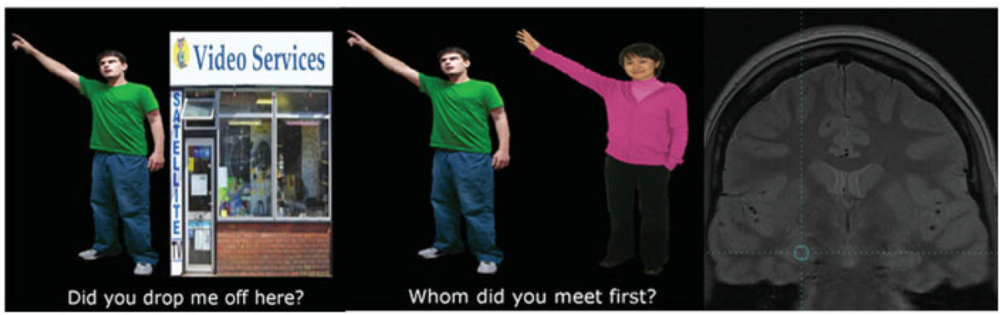

b.

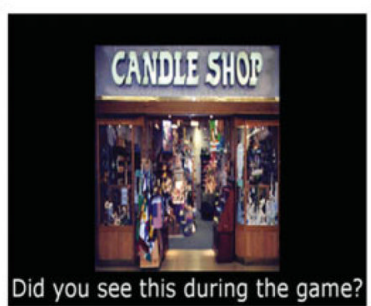

d.

FIGURE 1.

Methods and Electrode Placement. (a) Screen shot of a passenger waiting on sidewalk from a virtual city subjects explored. Following navigation, subjects performed retrieval involving: (b) item recognition, and (c) associative recognition (see methods). (d) Preimplant MRI showing placement of electrode targeting the right CA region of the anterior hippocampus of a patient-subject (MRI presented in radiological convention). Green target indicates area of microwires determined by coregistering post-implant CT with pre-implant MRI (CT electrode tracts minimized to improve clarity of anatomy). 
a. ERC, neuron1

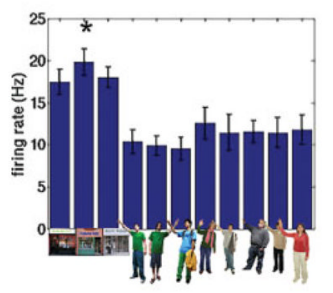

b. ERC, LFP 1

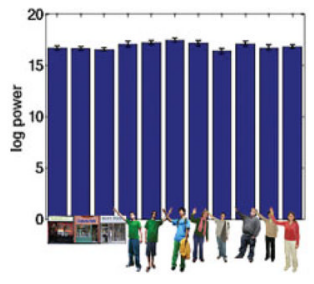

C. ERC, neuron 2

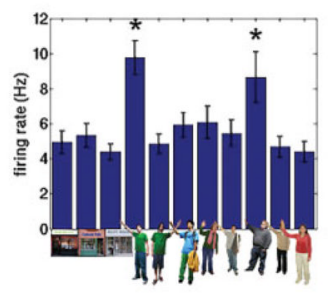

d. ERC, LFP 2

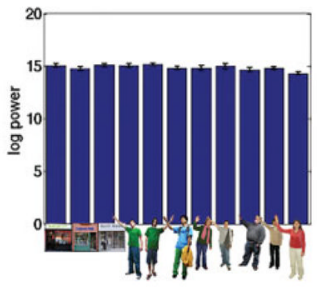

e. ERC, neuron 3

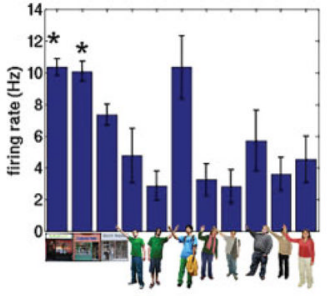

f. ERC, LFP 3

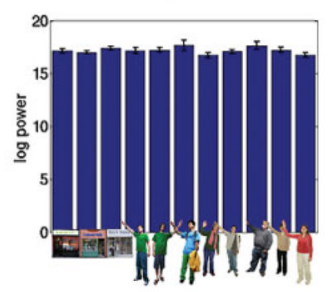

FIGURE 2.

Entorhinal Cortex Neurons Respond to Specific Items Regardless of Experimental Context. Neurons responded to specific items seen during navigation and continued firing throughout the experiment to these items during the memory condition. Top row $(\mathrm{a}, \mathrm{c}, \mathrm{e})$ shows the mean firing rate responses of three entorhinal cortex (ERC) neurons to items seen during navigation and retrieval; the item viewed is shown below each bar. All three ERC neurons showed significant effects of item in an ANOVA. Asterisks (*) show items for which the neurons were selective by comparing against all other items as revealed by Bonferroni corrected post-hoc $t$-tests; in general, ERC neurons responded to few items selectivity. Note that ERC neurons responded to both stores $(\mathrm{a}, \mathrm{e})$ and passengers (c), although we observed greater numbers of responses to stores in ERC overall (Figure $3 b$ ). Bottom row (b,d,f) shows the mean broadband log LFP power (decibels) on the same recording channel to each item. Theta-band and $\gamma$-band responses similarly did not show item-selectivity on these same channels. Only responses to items seen during navigation and retrieval are plotted. 
a.

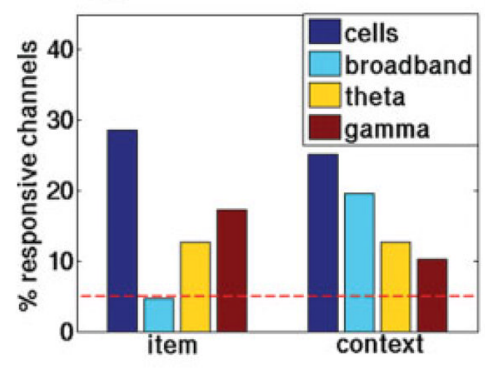

b.

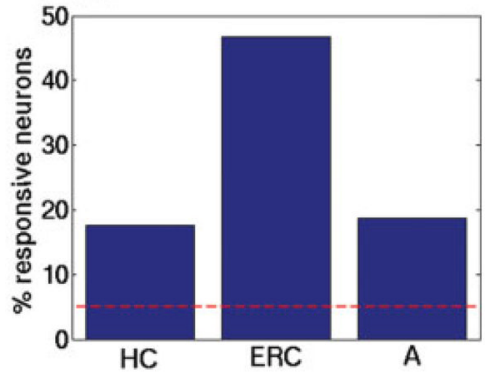

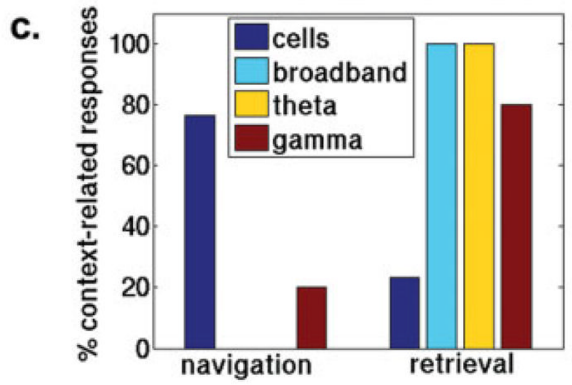

FIGURE 3.

Response Properties over the Population of Recorded Neurons and LFPs. (a) Neurons responded more to specific items (item) than they did to experimental context (context); while the broadband LFP responded to experimental context more than to items. Theta and $\gamma$-band LFPs responded significantly to items and context. Dotted red-line indicates Type I error rate of $P=0.05$. (b) The number of neurons responding significantly to viewing stores during navigation and retrieval were significantly greater in ERC compared with hippocampus (HC) or amygdala (A); $\chi^{2}(2)=6.2, P<0.05$. (c) When we further analyzed which specific experimental context (navigation or retrieval) cellular and LFP experimental context-responsive channels responded to, we found significantly more neurons responding during navigation than retrieval and significantly more LFPs (across all bands) responding during retrieval than navigation. 
a. ERC, neuron 1

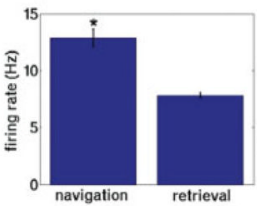

b. ERC, LFP 1

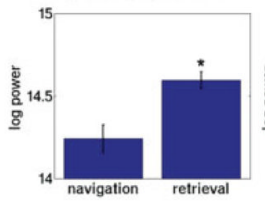

C. A, neuron 2

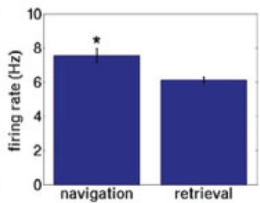

d. A, LFP 2
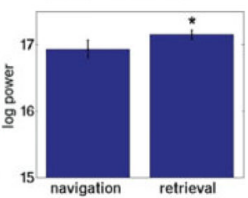

e. $\mathrm{HC}$, neuron 3

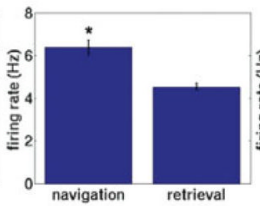

f. HC, LFP 3

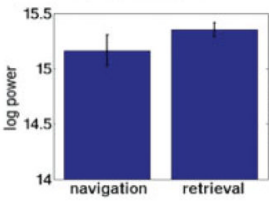

g. A, neuron 4

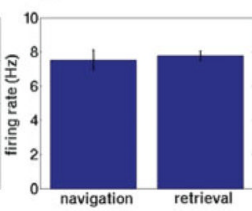

h. A, LFP 4

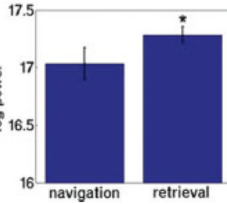

FIGURE 4.

MTL Neurons and LFPs Showed Significant Effects of Experimental Context. Significant numbers of neurons and LFPs responded significantly to experimental context. Neurons and LFPs, however, did not change in tandem with each other. The change in mean firing rate during navigation compared with retrieval for four MTL neurons is shown in a,c,e,g; neurons were recorded in right entorhinal cortex (ERC), right amygdala (A), right hippocampus (HC), and right amygdala, respectively, and from three different patients. Panels b,d,f,h show the mean log LFP power (decibels) on the same recording channels from which neurons were isolated ( $y$-axis on LFP plots is truncated so significant differences between conditions can be clearly visualized). Neurons a,c,e all showed greater firing during navigation, while LFP responses on these channels $(b, d, f)$ show increases during retrieval compared with navigation. [Color figure can be viewed in the online issue, which is available at www.interscience.wiley.com.] 


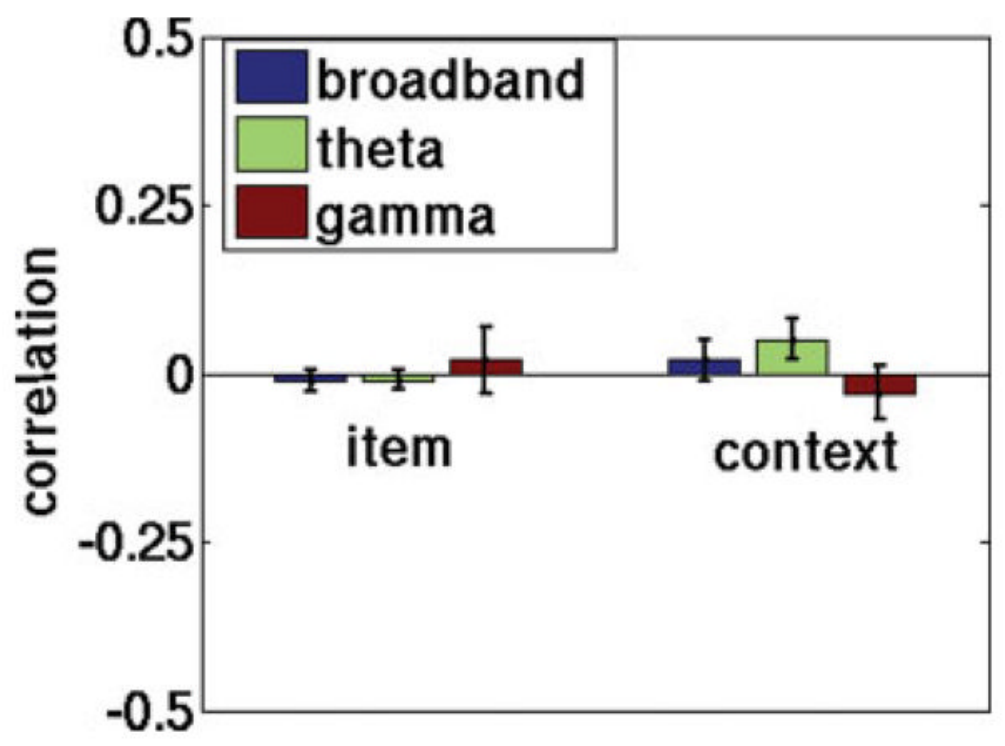

FIGURE 5.

No correlation between neural firing rate and wide-band, $\theta$-band, and $\gamma$-band LFPs. Mean correlation coefficient for cellular firing rate with wide-band $(1-100 \mathrm{~Hz}), \theta$-band $(4-8 \mathrm{~Hz})$, and $\gamma$-band wavelet-transformed LFP power $(40-100 \mathrm{~Hz})$ averaged over neurons that showed increased firing rate to specific items $(N=44)$ and neurons that showed increased firing for specific experimental contexts (encoding or retrieval: $N=30$ ). We observed no significant correlation between cellular firing rate and LFP power in wide-band, $\theta$-band, and $\gamma$-band power for items (items) and experimental context (context). 
